\title{
Generation of lossy mode resonances with absorbing thin-films
}

\author{
Ignacio Del Villar, Carlos R. Zamarreño, Miguel Hernaez, Francisco J. Arregui, Member, IEEE, and Ignacio R. \\ Matias, Senior Member, IEEE
}

\begin{abstract}
The generation of lossy mode resonances with absorbing thin-films is analyzed with electromagnetic theory. The main differences with surface plasmon resonances are presented and some rules are given towards an optimum design of sensing devices based on absorbing thin-film coated silica substrates. The material selected for the absorbing thin-film is ITO, which is adequate for supporting both surface plasmon resonances and lossy mode resonances.
\end{abstract}

Index Terms - Thin films, Indium Tin Oxide (ITO), electromagnetic theory

\section{INTRODUCTION}

$\mathrm{O}$ ptical waveguides coated with absorbing thin-films is a matter of research since many years both in the analysis of the physics [1-9], and in the development of sensors applications mainly focused on chemistry, biology and immunology [9-13]. Different types of modes can be supported by absorbing thin-films [7]. The most important resonance phenomenon caused by these modes is surface plasmon resonance (SPR). An SPR is generated by coupling between an evanescent wave and a surface plasmon [9]. However, there are other types of modes supported by absorbing thin films. Some authors call them guided modes [7], whereas others consider them as lossy modes [5,8]. Since lossy modes are a specific type of guided modes, it will be used henceforward the name "lossy modes" (LM). Consequently, the resonance caused by this type of mode will be called lossy mode resonance (LMR).

The number of applications based on each group of resonances has grown differently. There are tens of articles where SPR is used for sensing purposes, whereas the number of articles based on LMR is quite small. LMR based devices are used just for the determination of the thickness and permittivity of absorbing thin-films [7,14]. However, a promising work where it is proved for the first time its applicability as an optical fiber sensor [15], drives us to analyze the phenomenon and to obtain general rules towards a more efficient design of these devices.

To this purpose, LMRs are analyzed in detail with electromagnetic theory applied to the Kretschmann configuration [9]. It is indicated in [7] that a thin-film supports a lossy mode if the real part of its permittivity is positive and higher in magnitude than both its own imaginary part and the material surrounding the thin-film. The only necessary change that permits the film to support a surface plasmon is the real part of the thin film permittivity to be negative. In view of these conditions, transparent conductive oxides (TCO) are good candidates for supporting LMRs or SPRs thanks to the combination of conductive and transparency/reflectivity properties in the visible/infrared region respectively. In the region of high reflectance, the imaginary part of the TCO refractive index is of the order of metals [16,17]. Consequently, this region is adequate for SPR generation. However, for the low-reflectance region, the imaginary part is lower and permits the LMR generation. Observing both phenomena with the same material will help to understand, by contrast with SPR, the less explored characteristics of LMR.

Among TCO it has been selected Indium Thin Oxide (ITO) because it is one of the most popular ones. It is well know that TCO materials have been widely used in the fabrication of heat shields, liquid crystal displays, flat panel displays, organic light-emitting diodes, solar cells, and so forth $[16,18]$. More specifically, ITO has been also used in sensing applications such as the fabrication of conductimetric sensors [19]. In addition to this, the deposition of ITO on different substrates has been studied towards the development of optical sensors [20-23]. If all these good properties are joined to the fact that, as indicated above, the first LMR based optical sensor was developed with ITO coating [15], this justifies the utilization of ITO as the thin-film material for the results presented in this work.

The remainder of this paper is organized as follows. In Section 2 some remarks are indicated on the method used for the analysis of the propagation of light trough the thin-film, and on the modeling of ITO. In Section 3 numerical results are presented and some rules are given for the design of LMR based sensors. Conclusions are given in section 4 .

\section{THEORY}

In this section the models used for the simulations presented in section 3 are presented: the basic concepts for understanding the Kretschmann configuration, the model for the ITO layer and the model for the silica layer.

\section{A. Reflectance of Light in Kretschmann configuration}

The well known Kretschmann configuration is represented in Fig. 1. The material of the incident medium is silica because it is typically used as the substrate in many sensors. The material of the thin-film the light impinges onto is ITO. The surrounding dielectric medium (SDM) owns a refractive index of 1.321 (the refractive index of water at $1300 \mathrm{~nm}$ [24]). However, in the analysis done in section 3 several values of the SDM refractive index will be analyzed to see the sensitivity of the device to this parameter.
Manuscript received September 2, 2009. This work was supported in part by the Spanish Ministry of Education and Science-FEDER TEC2006-12170 and TEC2007-67987-C02-02/MIC.

The authors are with the Electrical and Electronic Engineering Department, Public University of Navarra, 31006 Pamplona, Spain (e-mail: ignacio.delvillar@unavarra.es). 


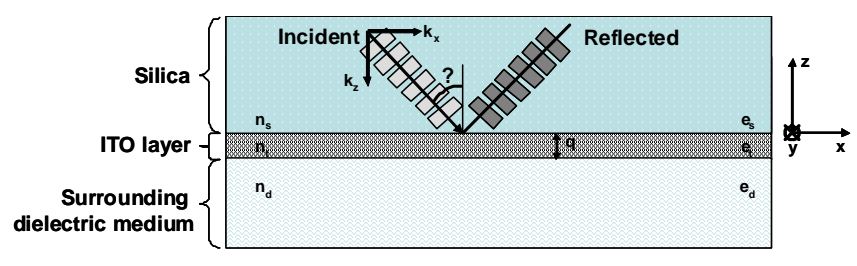

Fig. 1: Kretschmann configuration. Incident medium: silica, thin-film: ITO.

Assuming an incident plane wave and the silica - ITO - SDI infinite in the $\mathrm{x}-\mathrm{y}$ plane, the amplitude reflection coefficient can be obtained as [9]:

$$
r_{s t d}=\frac{r_{s t}+r_{t d} \exp \left(2 i k_{t z} q\right)}{1+r_{s t} r_{t d} \exp \left(2 i k_{t z} q\right)}
$$

where

$$
\begin{aligned}
& k_{i z}=\sqrt{\left(\frac{2 \pi}{\lambda}\right)^{2} \varepsilon_{i}-k_{x}^{2}} \\
& r_{i j}=\frac{\varepsilon_{j} k_{i z}-\varepsilon_{i} k_{j z}}{\varepsilon_{j} k_{i z}+\varepsilon_{i} k_{j z}} \text { for TM polarization } \\
& r_{i j}=\frac{k_{i z}-k_{j z}}{k_{i z}+k_{j z}} \text { for TE polarization } \\
& k_{x}=\left(\frac{2 \pi}{\lambda}\right) n_{s} \sin (\theta)
\end{aligned}
$$

and where subscripts $\mathrm{i}$ and $\mathrm{j}$ are $\mathrm{s}, \mathrm{t}$, or $\mathrm{d}$, according to Fig. 1 .

The reflectivity of the structure is expressed as the square of the absolute value of the amplitude reflection coefficient:

$R=\left|r_{s t d}^{2}\right|$

Another important parameter for the recognition of a resonance is the phase of $r_{\text {std }}$ [9], as it will be shown in section 3.

\section{B. ITO Layer}

Depending on the technique used for the deposition of ITO on optical fiber or any other substrate, the properties of the thin-film may differ in a great manner [16-18]. The most widely used expression for modeling of ITO is the Drude model [16,17]:

$$
\varepsilon(\omega)=\varepsilon_{\infty}-\frac{\omega_{p}^{2}}{\omega^{2}+i \frac{\omega}{\tau}}
$$

where $\varepsilon_{\infty}$ is the high frequency dielectric constant, $\tau$ is the electronic scattering time and $\omega_{\mathrm{p}}$ is the plasma frequency.

In [18] a more complex model is used, which leads to a better definition of the ITO refractive index. The formulation is expressed as follows:

$$
\varepsilon(\omega)=\varepsilon_{\infty}-\frac{\omega_{p}^{2}}{\omega^{2}+i \frac{\omega}{\tau}}+\frac{s_{0} \omega_{0}^{2}}{\omega_{0}^{2}-\omega^{2}-i \gamma \omega}
$$

where the additional term considers the dielectric behavior of
ITO. It is actually an oscillator model whose variables are: $\mathrm{s}_{0}$ (oscillator strength), $\omega_{0}$ (oscillator resonance frequency) and $\gamma$ (oscillator damping constant). The parameters used in that work are: $\varepsilon_{\infty}=3.57, \omega_{p}=1.89 \times 10^{15} \mathrm{rad} / \mathrm{s}, \tau=6.34 \times 10^{-15} \mathrm{~s} / \mathrm{rad}$, $s_{0}=0.49, \omega_{0}=5.61 \times 10^{15} \mathrm{rad} / \mathrm{s}$, and $\gamma=9.72 \times 10^{13}$. This parameterization leads to the curves of the refractive index and extinction coefficient that are represented in Fig. 2. It is easy to observe the two regions of high and low reflectivity indicated in the introduction. This model will be used henceforward for the numerical analysis.

\section{Silica core Layer}

As it was stated above, the incident medium is silica. The refractive index of fused silica can be estimated with the wellknown Sellmeier equation:

$n^{2}(\omega)=1+\sum_{j=1}^{m} \frac{B_{j} \omega_{j}^{2}}{\omega_{j}^{2}-\omega^{2}}$

With parameters: $\quad B_{1}=0.691663, \quad B_{2}=0.4079426$, $B_{3}=0.8974794, \lambda_{1}=0.0684043 \mu \mathrm{m}, \lambda_{2}=0.1162414 \mu \mathrm{m}$, and $\lambda_{3}=$ $9.896161 \mu \mathrm{m}$, where $\lambda_{j}=2 \pi c / \omega_{j}$ and $c$ is the speed of light in vacuum [25].

\section{NUMERICAL RESULTS}

By considering the evolution of the real part of the refractive index $n$ and the imaginary part of the refractive index $\mathrm{k}$ in the model represented in Fig. 2, it is easy to conclude that the reflective region of ITO is located above $2000 \mathrm{~nm}$, and the transparent region below $2000 \mathrm{~nm}$. According to the conditions given in the introduction, SPRs should be supported by the ITO layer for wavelengths above $2000 \mathrm{~nm}$ (the real part of the permittivity is negative), whereas LMRs should be supported for wavelengths below $2000 \mathrm{~nm}$.

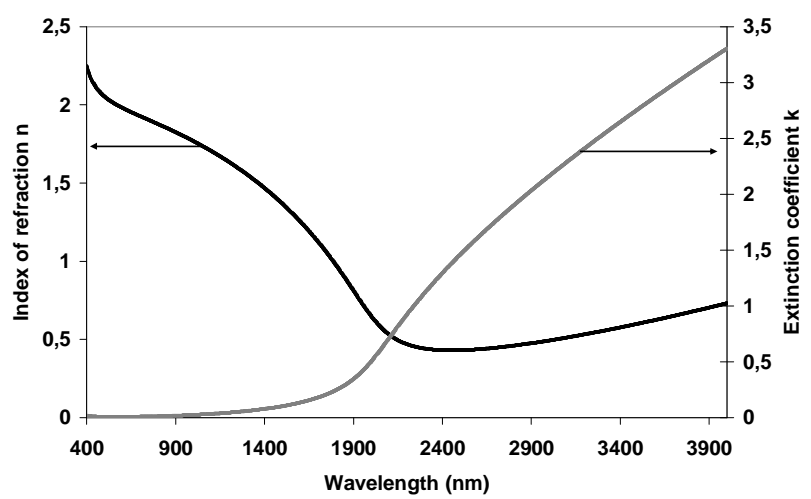

Fig. 2: ITO model. Index of refraction $\mathrm{n}$ and extinction coefficient $\mathrm{k}$ of ITO deposited layer

To prove the previous assumption, a Kretschmann configuration is analyzed with silica as the incident medium, an SDI refractive index of 1.321, which is the refractive index of water at $1300 \mathrm{~nm}$ [24], and an ITO layer of thickness 100 $\mathrm{nm}$. The reflectance for different angles of incidence ranging from 80 to 89 degrees is presented in Fig. 3 both for TE and TM polarization. 

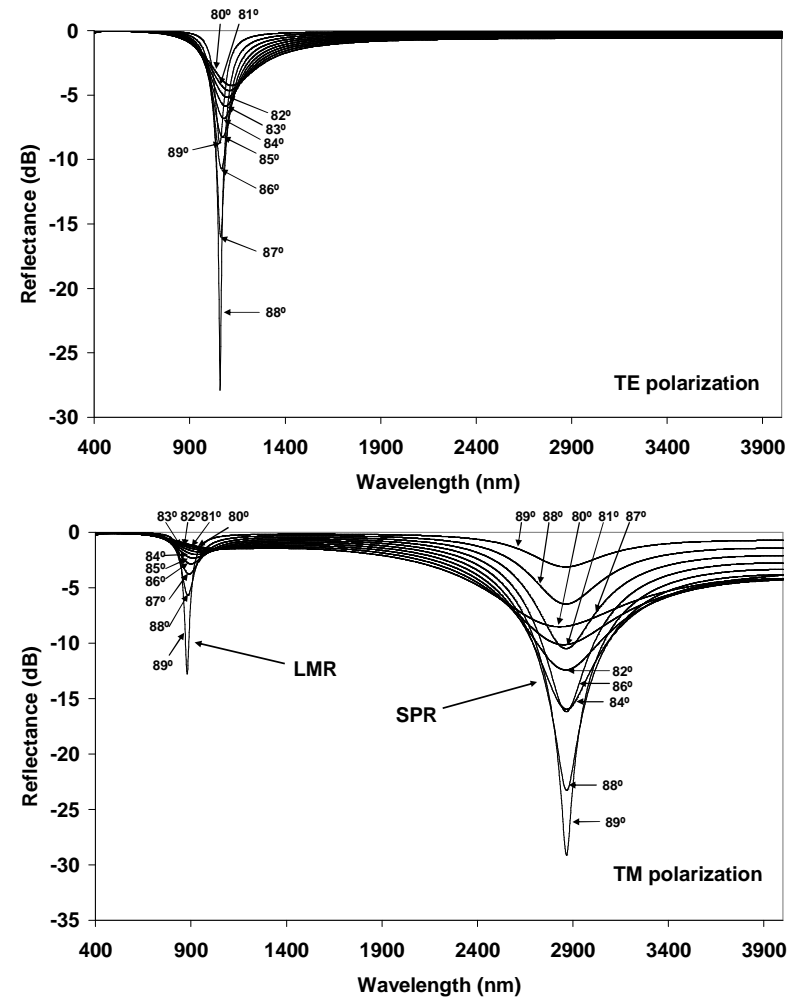

Fig. 3: Reflectance spectrum $(\mathrm{dB})$ for different angles of incidence for an ITO thin-film of 100nm: a) TE polarization; b) TM polarization

Two resonances can be observed: one of them is located at around $2500 \mathrm{~nm}$ and is only visible for TM polarization, whereas the other one is located at about $1000 \mathrm{~nm}$ and is visible both for TE and TM. These results are in agreement with works developed elsewhere [7-9], where SPRs can only be generated if the real part of the permittivity is negative for TM polarization, and LMRs can be generated if the real part of the permittivity is positive both for TE and TM polarization. SPRs are wider that LMRs. However, this is caused by the different dispersion of the silica substrate in the left and the right side of the spectrum analyzed. Furthermore, several LMRs can be obtained experimentally with $\mathrm{TiO} 2$ coatings. In this case LMRs located at large wavelengths are also wider than LMRs located at short wavelengths.

In order to see a more evident prove of the different nature of LMRs and SPRs, it can be observed in Fig. 4 the results obtained by setting an ITO layer much thicker $(1000 \mathrm{~nm})$ than in Fig. 3. The result is that no SPR is visible [27]. It is well known that an SPR has an optimum thickness where there is a maximum light coupling. As the ITO thickness increases, the resonance moves to higher wavelengths and there is a less significant coupling. This is not the case for an LMR. In fact more LMRs are created in Fig. 4.

Once the different nature of LMRs and SPRs has been observed, focus will be centered on explaining the generation of an LMR. In [2] it is explained the coupling that occurs in a planar waveguide coated with a thin-film. There is a periodical coupling between a mode in the waveguide and the lossy modes in the thin-film. This behavior can also be observed in a cylindrical waveguide [28]. The conditions for a maximum coupling are mainly two: there is a considerable overlap between the mode fields, and the phase-matching condition (i.e. the equality of real parts of propagation constants) is sufficiently satisfied [5]. Since the phenomenon occurs when the lossy mode is near cut-off, it is stated in [2] that there are cut-off thickness values that lead to attenuation maxima. In other works similar conclusions are extracted after a thorough analysis of the modes. As the thickness of the thin-film on the waveguide is increased, some modes guided in the optical waveguide become guided in the thin-film, which causes a modal redistribution or modal conversion $[6,28]$.
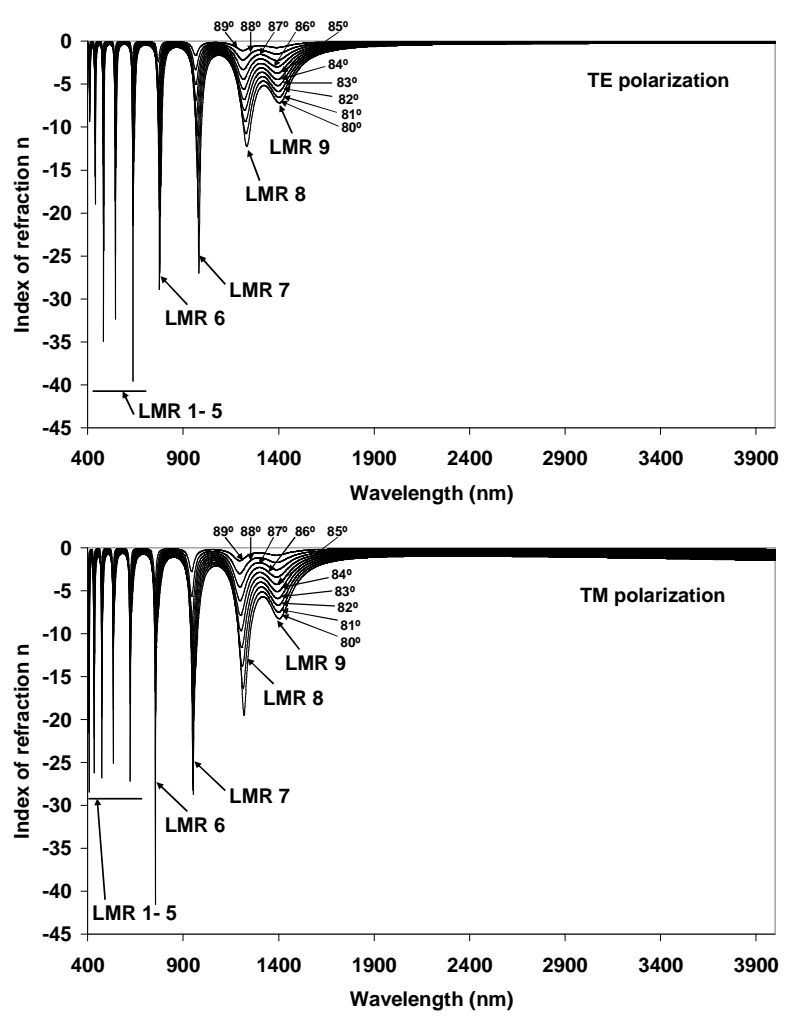

Fig. 4: Reflectance spectrum (dB) for different angles of incidence for an ITO thin-film of $1000 \mathrm{~nm}$ : a) TE polarization; b) TM polarization

However, in the previous studies the Kretschmann configuration was not used. Consequently, the analysis of the effect of the angle of incidence was not possible. Now a similar analysis is done with the model presented in section 2, which accounts for the influence of the angle of incidence. For the same angles used in Fig. 3 and Fig. 4, it is represented in Fig. 5 the absorbance as a function of the ITO thickness.

Again there are absorbance maxima at specific thickness values. Nonetheless, it is easy to appreciate that the position of the maxima is not the same for all angles. In fact, the position of the various LMRs of Fig. 3 and Fig. 4 is also dependent on the angle of incidence. Moreover, the depth of the resonance is also dependent on the angle of incidence. These questions can be better understood if the effective indices of modes guided in the ITO region are analyzed (see Fig. 6). The same parameters of Fig. 4 will be used. On the one hand, in Fig. 6 
the effective indices of the lossy modes (LM), cross the plot of the core refractive index approximately at the wavelengths of the various LMR in Fig. 4. On the other hand, it is stated in [10] that the effective index of the evanescent waves in the thin-film can be expressed as:

$$
n_{\text {eff }}=n_{s} \sin (\theta)
$$

where $\mathrm{n}_{\mathrm{s}}$ is the silica substrate refractive index and $\theta$ is the angle of incidence.
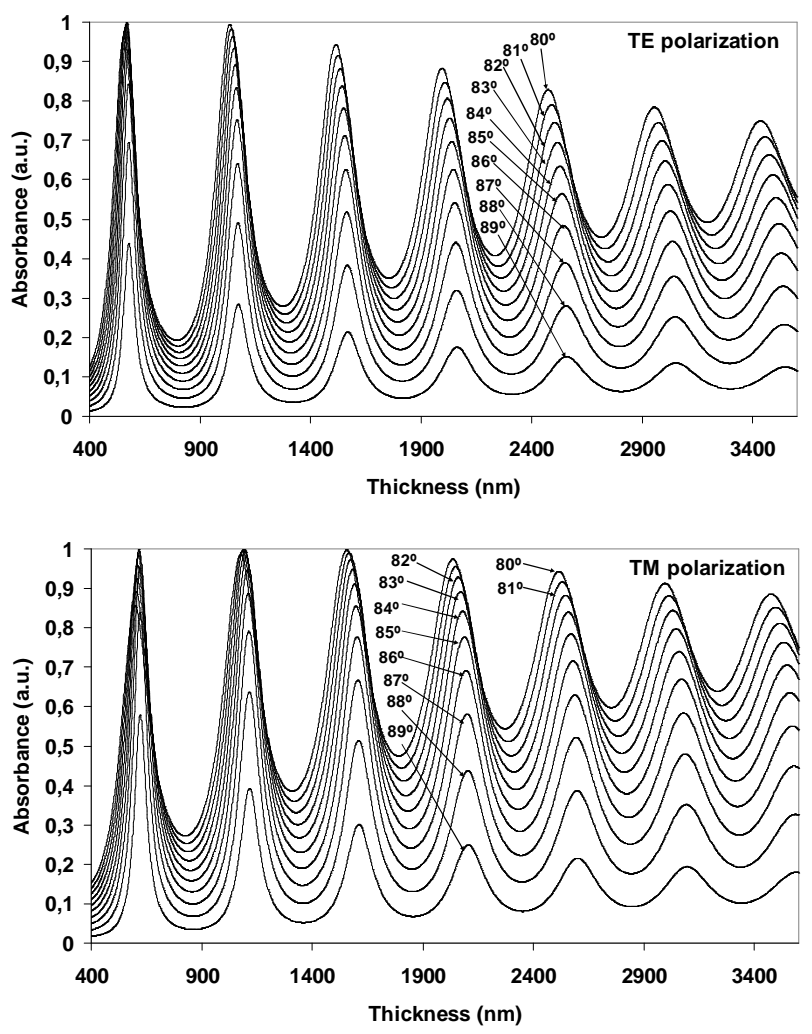

Fig. 5: Reflectance (dB) for different angles of incidence as a function of ITO thin-film thickness: a) TE polarization; b) TM polarization

It is well known that for the generation of an LMR a coupling between an evanescent wave and a surface wave must occur. Now there is a coupling between an evanescent wave and a lossy mode guided in the thin-film.

If the angle is $90^{\circ}$ the effective index of the evanescent wave is $n_{\mathrm{s}}$. As well as the angle decreases, the effective index of the corresponding evanescent wave decreases. Since an LMR is caused by coupling between an evanescent wave and a lossy mode, and in Fig. 6 lossy modes decrease in effective index as the wavelength increases, coupling must be shifted to higher wavelengths as the angle of incidence decreases.

In order to illustrate the previous explanation, two LMRs are selected (located at 1000 and $1300 \mathrm{~nm}$ ) and it is analyzed the reflectance for a wide range of incidence angles in Fig. 7.
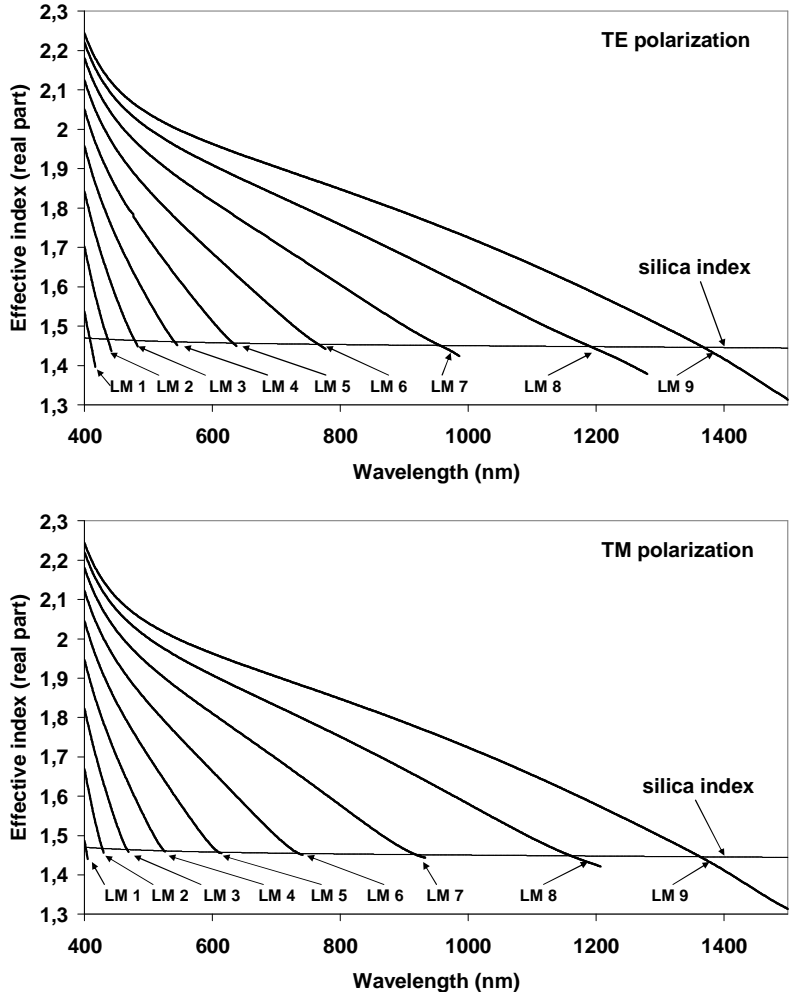

Fig. 6: Effective index of modes for an ITO thin-film thickness $1000 \mathrm{~nm}$ as a function of wavelength: a) TE polarization; b) TM polarization
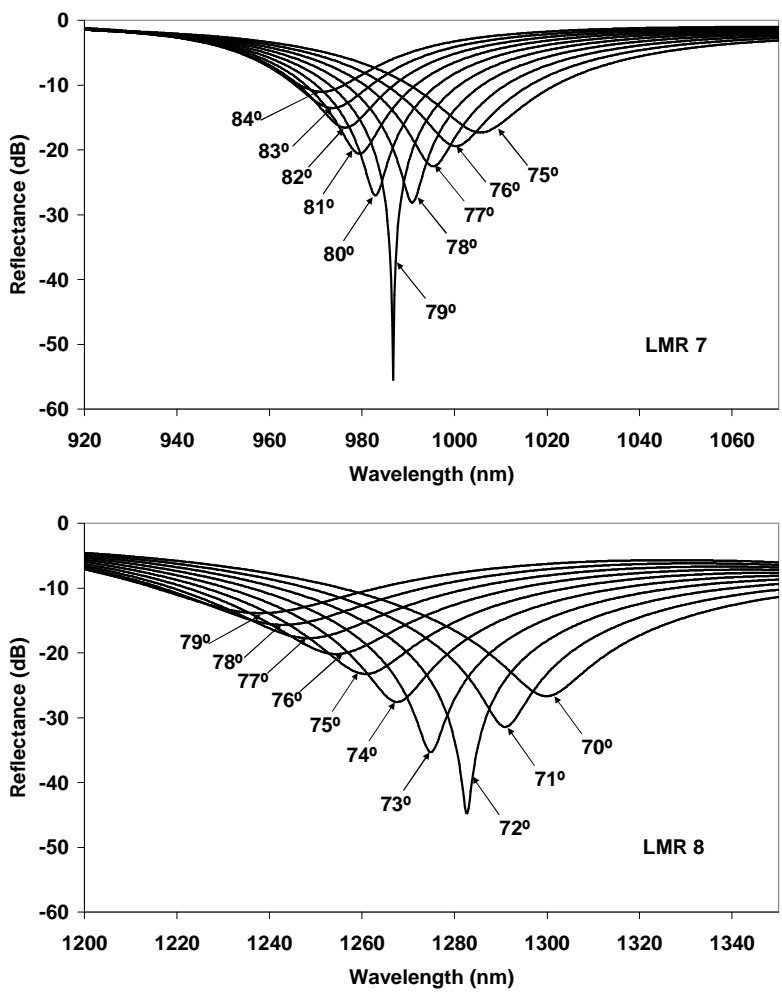

Fig. 7: Reflectance spectra for different angles of incidence (TE polarization): a) LMR 7 of Fig. 4; b) LMR 8 of Fig. 4

For the sake of simplicity only the results for TE 
polarization are presented. In both cases the resonance is shifted to higher wavelengths as the angle of incidence decreases. It can also be visualized that for the band located at about $1000 \mathrm{~nm}$ a maximum at $79^{\circ}$ is observed, whereas for the band at $1300 \mathrm{~nm}$ the maximum is shifted to $72^{\circ}$. Moving back to the results of Fig. 6, the mode that is responsible for the generation of the LMR at about $1000 \mathrm{~nm}$ is no longer guided at a specific wavelength. The highest wavelength where this mode is guided is $986.73 \mathrm{~nm}$. At this wavelength the effective index of the lossy mode is 1.4239 and the substrate index is 1.4506. The angle that satisfies this condition in expression (10) is $78.99^{\circ}$. The same operation is valid for the LMR at $1300 \mathrm{~nm}$. In this case the wavelength is $1280.59 \mathrm{~nm}$, the effective index of the mode is 1.3783 and the substrate index is 1.471 . The result is an angle of $72.26^{\circ}$.

The explanation is that there is a wavelength value when a lossy mode starts to be guided in the ITO region. At this specific wavelength, the effective index of an evanescent wave coincides with the effective index of a lossy mode. Consequently, there is a maximum overlap between the mode fields and a maximum transfer of energy [5]. As the lossy mode increases its effective index and is more confined to the thin-film, the effective indices of the evanescent wave and the lossy mode start to be different, and the coupling is not maximum. This is the reason why for the rest of angles there is a decrease in the resonance depth.

The analysis of the phase also helps to understand the LMR. It is well known that the interaction between an incident light and a surface plasmon affects the phase of the reflected light, which exhibits an abrupt jump at the wavelength range where the resonance occurs [29]. This is also true for an LMR, as it can be observed in the analysis of Fig. 8, which is done for the same parameters of Fig. 7. In other words, Fig. 7 and Fig. 8 represent the reflectivity and the phase as a function of the wavelength respectively.

In Fig. 8 there are two types of evanescent waves: those generated with an incidence angle below the angle of maximum coupling and those generated with an incidence angle above the angle of maximum coupling. Apparently it seems that there is a discontinuity for those angles above the angle of maximum coupling. However, it is just the pass from $180^{\circ}$ to $-180^{\circ}$. After this explanation, it is easy to appreciate that as the angle of maximum coupling is approached from both groups of evanescent waves, the phase change is more abrupt. The most abrupt change for both groups of evanescent waves occurs for the angle which is close to the angle of maximum coupling. In Fig. 8a this occurs for angles $78^{\circ}$ and $79^{\circ}$ (the angle of maximum coupling is $78.99^{\circ}$ ) and in Fig. $8 \mathrm{~b}$ this occurs for angles $72^{\circ}$ and $73^{\circ}$ (the angle of maximum coupling is $72.26^{\circ}$ ). An analogy can be made with the phenomenon observed in a cylindrical waveguide [28], where it was proved that there are modes with effective index above the effective index of the mode that is guided in the thin-film which "compete" for being guided in the thin film. Once a mode is guided in the thin-film, these modes recover their original state.
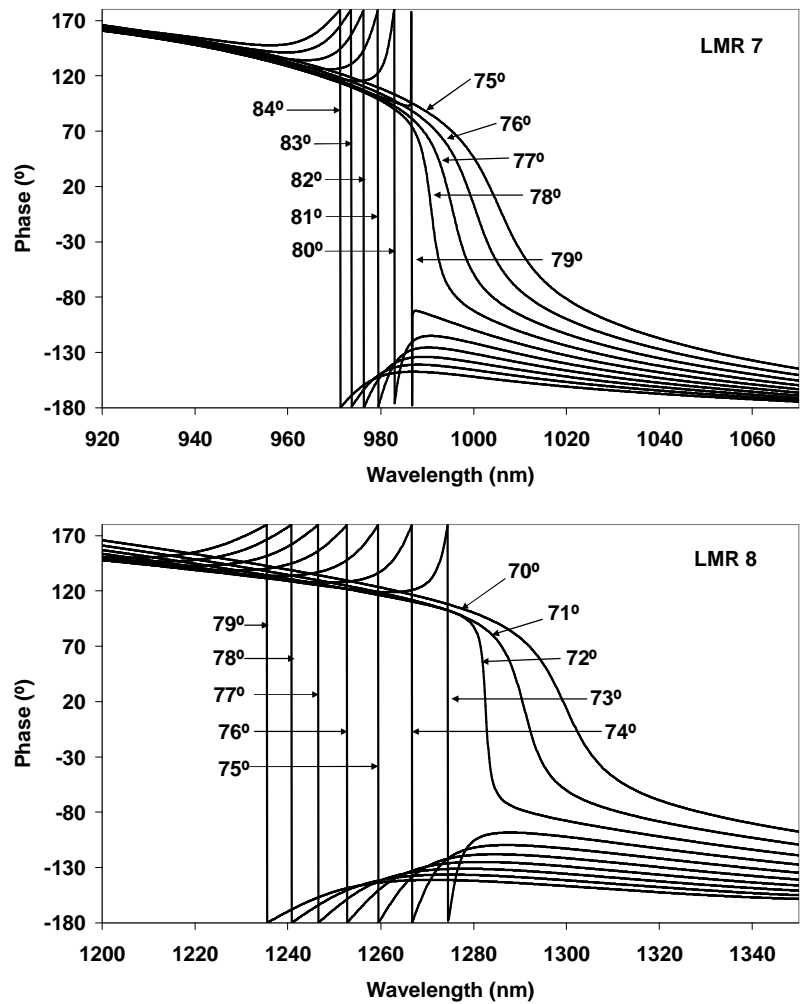

Fig. 8: Phase spectra for different angles of incidence: a) LMR 7 of Fig. 4; b) LMR 8 of Fig. 4

In Fig. 8 those evanescent waves generated with angles of incidence above the angle of maximum coupling can be considered with higher effective index. The phase of the reflected light phase shifts from $180^{\circ}$ to $0^{\circ}$. However, once a mode is guided in the thin-film, the reflected light phase moves back to the original state of $180^{\circ}$. On the other hand, for those evanescent waves generated with angles of incidence below the angle of maximum coupling there is a complete phase shift from 180 to $-180^{\circ}$. The phase shift is more abrupt for angles closer to the angle of maximum coupling. This last question has also an effect in the width of the resonance. For those angles that induce a less abrupt phase change, the resonance is wider (see Fig 7).

Finally, it is important to analyze the effect of thickness in the design of an LMR based device. It is obvious that the central wavelength of the LMR can be tuned by using a different parameterization of the ITO layer. If a different ITO model such as that of [16] is used, the LMR will move to lower wavelengths. The reason is that the wavelength where the real part of the permittivity starts to be negative is located at about $1400 \mathrm{~nm}$. Consequently, no LMR will be located at wavelengths above $1400 \mathrm{~nm}$. However, another way of selecting the position of the LMR in the electromagnetic spectrum is by setting the ITO thickness. In view of the results observed in Fig. 3 and Fig. 4, it is clear that the change in the ITO thickness is responsible for the number of LMRs in the electromagnetic spectrum. The increase of thickness permits to generate several lossy modes in the ITO thin-film and, consequently, to create more wavelength ranges where 
coupling of energy to lossy modes exist. However, it is important to know about the relation between the evolution of modes as a function of wavelength and the sensitivity of the device. To this purpose two different LMRs will be analyzed: the LMR 1 and the LMR 8 of Fig. 4. The purpose of selecting these bands is to observe the sensitivity in two resonances induced by coupling to lossy modes with a different evolution. In Fig. 6 the lossy mode responsible for the generation of the LMR 1 has an abrupt change as a function of wavelength, whereas the one responsible for the LMR 8 has a less abrupt change. In Fig. 9a and Fig. 9b, it is analyzed the spectra for different surrounding dielectric media which correspond with concentrations of glycerol in water: $1.321(0 \%), 1.339(15 \%)$, $1.358(30 \%)$, and $1.378(45 \%)$ [30,31]. For both LMR the incidence angle was $80^{\circ}$. The result is that for LMR 1 the sensitivity is $11.05 \mathrm{~nm} / \mathrm{RIU}$ (RIU = refractive index unit), which is much lower than the sensitivity of the LMR 8 (233.33 nm/RIU).
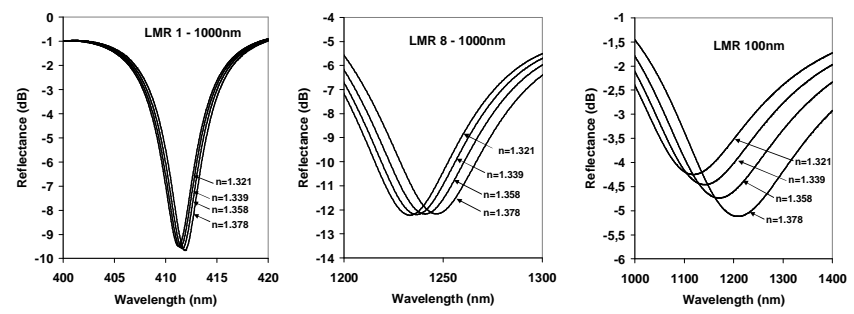

Fig. 9: Reflectance spectra (TE polarization) for different surrounding medium refractive indices: $1.321,1.339,1.358$ and 1.378: a) LMR 1 of Fig. 4; b) LMR 8 of Fig. 4; c) LMR of Fig. 3.

Another way to increase the sensitivity is to select a narrower thin-film. In this way there are fewer lossy modes and the change as a function of wavelength is not as abrupt as when there are more lossy modes. To prove this fact it is analyzed the sensitivity for a thin-film of $100 \mathrm{~nm}$ in fig. 9c. The result is a sensitivity of $1578.95 \mathrm{~nm} / \mathrm{RIU}$. This value is comparable with the sensitivity of SPR sensors, which range from 1000 to 10000 [32], and can even be improved with other coating materials such as $\mathrm{TiO} 2$ [26]. The main conclusion of these last results is that the sensitivity is increased if the variation of the effective index of the lossy mode as a function of wavelength is reduced.

\section{CONCLUSION}

In this work it has been analyzed the generation of lossy mode resonances (LMR) with electromagnetic theory based on the Kretschmann configuration. The incidence medium was silica. In order to understand the conditions for LMR generation, an absorbing thin-film of Indium Tin Oxide (ITO) has been used for coating the silica substrate. This material supports both surface plasmon resonances (SPR) and LMRs. As a result it was possible to see the differences between both phenomena: an SPR is only visible for TM polarization whereas an LMR is visible for both polarizations. It is possible to generate several LMRs if the absorbing thin-film thickness is increased, which is not possible for SPRs. The phase and the module of the absolute reflection coefficient are critical for the generation of an LMR. In fact, for a specific angle of incidence the maximum attenuation of the LMR occurs when the phase experiments an abrupt change.

In terms of mode coupling theory, an LMR is the result of light coupling between evanescent waves and lossy modes guided in the absorbing thin-film. Consequently, the analysis of the evolution of the modal effective index permits to understand the generation of the resonance. Moreover, the less or more abrupt change of the effective index as a function of the incidence wavelength leads to higher or lower sensitivity if it is used as a sensor. As an example it has been studied the response of the sensor against different surrounding medium refractive indices. The result was that the sensitivity increases if the variation of the modal effective index is less abrupt. This occurs for high order LMRs and if there are few lossy modes supported by the thin film. In other words, the thickness of absorbing thin-films is critical for obtaining a highly sensitive device. In view of the interesting properties of LMRs, more applications should be found in other fields such as optical communications.

\section{ACKNOWLEDGMENT}

The authors acknowledge financial support to the Spanish Ministry of Education and Science-FEDER TEC2009-09210 and TEC2007-67987-C02-02/MIC.

\section{REFERENCES}

[1] D. Sharid, "Long-range surface-plasma waves on very thin metal-films," Phys. Rev. Lett., vol. 47, pp. 1927-1930, 1981.

[2] G. M. McWright, T. E. Batchman, and M. S. Stanziano, "Measurement and analysis of Periodic Coupling in Silicon-Clad Planar Waveguides," IEEE Trans. on MTT, vol. 30, pp. 1753-1759, 1982.

[3] M. O. Petersen and B. S. Zhu, "Dielectric function of metals determined by surface-plasmon excitation: ambiguities caused by ultrathin oxide layers," J. Opt. Soc. Am. A, vol. 5, pp. 471-474, 1988.

[4] F. Yang, J. R. Sambles, and G. W. Bradberry, "Long-range coupled surface exciton polaritons,” Phys. Rev. Lett.., vol. 64, pp. 569-562, 1990.

[5] M. Marciniak, J. Grzegorzewski and M. Szustakowski, "Analysis of lossy mode cut-off conditions in planar waveguides with semiconductor guiding layer," IEE Proceedings J., vol. 140, pp. 247-251, 1993.

[6] R. F. Carson and T. E. Batchman, "Multimode phenomena in semiconductor-clad dielectric optical waveguide structures," App. Opt., vol. 29, pp. 2769-2780, 1990.

[7] F. Yang and J. R. Sambles, "Determination of the optical permittivity and thickness of absorbing films using long range modes," J. Mod. Opt., vol. 44, pp. 1155-1163, 1997.

[8] D. Razansky, P. D. Einziger and D. R. Adam, "Broadband absorption spectroscopy via excitation of lossy resonance modes in thin films," Phys. Rev. Lett., vol. 95, pp. 018101, 2005.

[9] J. Homola, "Surface Plasmon Resonance Based Sensors" (Springer, 2006).

[10] R. C. Jorgenson and S. S. Yee, "A fiber-optic chemical sensor based on surface Plasmon resonance," Sens. Actuators B, vol. 12, pp. 213-220, 1993.

[11] D. Monzón-Hernández, J. Villatoro, D. Talavera, and D. Luna-Moreno, "Optical-Fiber Surface-Plasmon Resonance Sensor with Multiple Resonance Peaks,” App. Opt., vol. 43, pp. 1216-1220, 2004.

[12] R. Slavik, J. Homola and R. Brynda, "A miniature fiber optic surface plasmon resonance sensor for fast detection of staphylococcal enterotoxin B," Bionsens. Bioelectron., vol. 17, pp. 591-595, 2002.

[13] C. D. Chen, S. F. Cheng, L. K. Chau, and C. R. C. Wang, "Sensing Capability of the Localized Surface Plasmon Resonance of Gold Nanorods," Biosens. Bioelectron., vol. 22, pp. 926-932, 2007. 
[14] K. S. Chiang, S. Y. Cheng, and Q. Liu "Characterization of Ultrathin Dielectric Films with the Prism-Coupler Method," J. Lightwave Technol., vol. 25, pp. 1206-1212, 2007.

[15] C. R. Zamarreño, M. Hernaez, I. Del Villar, I. R. Matias, and F. J. Arregui, "ITO Coated Optical Fiber Refractometers Based on Resonances in the Infrared Region," IEEE Sens. J., vol. 10, pp. 365-366, 2010.

[16] I. Hamberg, A. Hjortsberg, and C. G. Granqvist, "High quality transparent heat reflectors of reactively evaporated indium tin oxide," Appl. Phys. Lett., vol. 40, pp. 362-364, 1982.

[17] S. H. Brewer, and S. Franzen, "Optical properties of indium tin oxide and fluorine-doped tin oxide surfaces: correlation of reflectivity, skin depth, and plasmon frequency with conductivity," J. Alloys and Compounds, vol. 338, pp. 73-79, 2002.

[18] S. Laux, N. Kaiser, A. Zöller, R. Götzelmann, H. Lauth and H. Bernitzki, "Room-temperature deposition of indium tin oxide thin films with plasma ion-assisted evaporation" Thin Solid Films, vol. 335, pp. 1$5,1998$.

[19] N. G. Patel, P. D. Patel and V. S. Vaishnav, "Indium tin oxide (ITO) thin film gas sensor for detection of methanol at room temperature," Sens. Actuators B, vol. 96, pp. 180-189, 2003.

[20] B. J. Luff, J. S. Wilkinson and G. Perrone, "Indium tin oxide overlayered waveguides for sensor applications," App. Opt., vol. 36, pp. 7066-7072, 1997.

[21] T. Konry, and R. S. Marks, "Physico-chemical studies of indium tin oxide-coated fiber optic biosensors," Thin Solid Films, vol. 492, pp. 313 $321,2005$.

[22] T. Konry, A. Novoa, S. Cosnier, and R. S. Marks, "Development of an "Electroptode" immunosensor: Indium tin oxide-coated optical fiber tips conjugated with an electropolymerized thin film with conjugated cholera toxin B subunit," Anal Chem., vol. 75, pp. 2633-2639, 2003.

[23] O. Salama, S. Herrmanna, A. Tziknovskyb, B. Piurac, M. Meirovichc, I Trakhtd, B. Reede, L. I. Lobelb, and R. S. Marksa "Chemiluminescent optical fiber immunosensor for detection of autoantibodies to ovarian and breast cancer-associated antigens," Biosens. Bioelectron., vol. 22, pp. 1508-1516, 2007.

[24] D. Masahiko, and A. Masumura, "Measurement of the refractive index of distilled water from the near infrared to the ultraviolet region," App. Opt., vol. 46, pp. 3811-3820, 2007.

[25] I. H. Malitson, "Interspecimen Comparison of the Refractive Index of Fused Silica," J. Opt. Soc. Am., vol. 55, pp. 1205-1208, 1965.

[26] M. Hernaez, I. Del Villar, C. R. Zamarreño, F. J. Arregui and I. R. Matias "Optical fiber refractometers based on lossy mode resonances supported by TiO2 coatings," Appl. Opt., vol. 44, pp. 3980-3985, 2010.

[27] Del Villar, C. R. Zamarreño, M. Hernaez, F. J. Arregui and I. R. Matias, "Multiple-peak Resonances with Indium Tin Oxide Coated Optical Fibers", J. Lightwave Technol., vol. 28, no. 1, pp. 111-117, 2010.

[28] I. Del Villar, I. R. Matias, F. J. Arregui and M. Achaerandio, "Nanodeposition of materials with complex refractive index in longperiod fiber gratings," J. Lightwave Technol., vol. 23, pp. 4192-4199, 2005.

[29] R. T. Deck, D. Sarid, G. A. Olson, and J. M. Elson, "Coupling between finite electromagnetic beam and long-range surface plasmon mode" App. Opt., vol. 22, 3397-3405, 1983.

[30] P. R. Cooper, "Refractive index measurements of liquids used in conjunction with optical fiber," App. Opt. vol. 22, pp. 3070-3072, 1983.

[31] L. F. Hoytt, "New Table of the Refractive Index of Pure Glycerol at $20^{\circ}$ C," Ind. Eng. Chem., vol. 26, pp. 329-332, 1934.

[32] B. Lee, S. Roh, and R. J. Park, "Current status of micro- and nanostructured optical fiber sensors," Opt. Fiber Technol., vol. 15, pp. 209$221,2009$.

Ignacio Del Villar received his MS degree in Electrical and Electronic Engineering and his Ph.D. degree, speciality in Optical Fiber Sensors in 2002 and 2006, respectively from the Public University of Navarra (UPNA).

During 2004 was a visiting scientist at the Institute d'Optique (Orsay, France) and in 2005 he was a visiting scientist at the Applied Physics Departament of the University of Valencia (Burjassot, Spain). He is presently a lecturer at the Public University of Navarra.

His research interest includes optical fiber sensors and the analysis of waveguides and nanostructured materials.

Carlos R. Zamarreño received the MS degree in Electrical and Electronic Engineering and his $\mathrm{Ph}$. D in Communications from the Public University of Navarra (UPNA) in 2005 and 2009 respectively. During 2008 he was a visiting scientist at the Massachusetts Institute of Technology (Boston,MA, USA) and he has been working as an associate researcher at the UPNA since 2007. He is currently working as an associate lecturer at the UPNA and his main research interests are optical fiber sensors, passive optical devices and their engineering applications.

Miguel Hernaez received the M.S. degree in Telecommunications Engineering from the Public University of Navarra (UPNA), Spain, in 2008. In 2009, he finished the Master degree in Communications. He is currently working towards the Ph.D. degree in the Communications Doctoral Program in the Electrical and Electronic Engineering Department, UPNA.

His research interests include optical fiber sensors and nanostructured materials.

Francisco J. Arregui is an Associate Professor at the Public University of Navarre, Pamplona, Spain. He was part of the team that fabricated the first optical fiber sensor by means of the layer-by-layer self-assembly method at Virginia Tech, Blacksburg, VA, USA, in 1998.

$\mathrm{He}$ is the author of around 250 scientific journal and conference publications, most of them related to optical fiber sensors based on nanostructured coatings. Francisco J. Arregui is an Associate Editor of the Journals "IEEE Sensors Journal" and "International Journal on Smart Sensing and Intelligent Systems" as well as Editor-in-Chief of "Journal of Sensors". $\mathrm{He}$ is also the editor of the book "Sensors based on nanostructured materials" which has been recently published.

Ignacio R. Matias received his MS degree in Electrical and Electronic Engineering and his Ph.D. degree, speciality in Optical Fiber Sensors in 1992 and 1996, respectively from the Polytechnic University of Madrid (UPM), Spain. In 1996 he took up a lectureship at the Public University of Navarra (Pamplona, Spain) where presently he is a Permanent Professor.

He has co-authored hundreds of journal and conference papers related to optical fiber sensors, passive optical devices and systems. He is an IEEE Senior member. He is a Senior Editor of the IEEE Sensors Journal. 